\title{
Phase-sensitive Manipulations of Squeezed Vacuum Field in an Optical Parametric Amplifier inside an Optical Cavity
}

\author{
Jing Zhang ${ }^{1, \dagger}$, Chenguang $\mathrm{Ye}^{1}$, Feng $\mathrm{Gao}^{1}$, Min Xiao ${ }^{1,2}$ \\ ${ }^{1}$ The State Key Laboratory of Quantum Optics and Quantum Optics Devices, \\ Institute of Opto-Electronics, Shanxi University, Taiyuan 030006, P.R. China and \\ 2 Department of Physics, University of Arkansas, Fayetteville, Arkansas 72701, USA
}

\begin{abstract}
Squeezed vacuum field can be amplified or deamplified when it is injected, as the signal beam, into a phase-sensitive optical parametric amplifier (OPA) inside an optical cavity. The spectral features of the reflected quantized signal field are controlled by the relative phase between the injected squeezed vacuum field and the pump field for the OPA. The experimental results demonstrate coherent phenomena of OPA in the quantum regime, and show phase-sensitive manipulations of quantum fluctuations for quantum information processing.
\end{abstract}

Manipulations of quantum states of light, during their propagation, storage, and frequency conversion, are essential for quantum information processing and quantum networking. Recent experiments have demonstrated the transmission [1], slowing down [2, 3, 4], and storage and retrieval [5, [6] of squeezed states of light through electromagnetically induced transparency in multi-level atomic systems [7], which are important to implement quantum network protocol [8, 9]. Another important aspect in manipulating quantum fluctuations is the phase-sensitive amplification and deamplification of the squeezed states of light, which are needed in quantum information propagation and communication. An optical parametric amplifier can be used to amplify non-classical states such as squeezed states and single photon states. This process, referred to as the "quantum injected optical parametric amplification", turns out to be particularly fruitful in the implementations of discrete-variable and continuousvariable $(\mathrm{CV})$ quantum information processing, such as optimal quantum cloning machines [10, 11], optical quantum U-Not gate [12], bridge between "microscopic" and "macroscopic" entanglement [13], and CV all-optical quantum teleportation [14]. Bruckmeier, et al experimentally demonstrated that improved quantum nondemolition measurements could be realized by injecting amplitude-squeezed light into the meter input port of an optical parametric amplifier (OPA) [15]. Recently, Agarwal [16] has theoretically studied the phase-sensitive responses of quantum states of light through an OPA inside an optical cavity operated below threshold [17]. Spectral splitting due to quantum interferences between the input quantum field and the generated down-converted subharmonic field has been predicted [16].

In this Letter, we experimentally demonstrate such quantum interference phenomena in the phase-sensitive OPA system inside an optical cavity with an injected squeezed vacuum state. Previously we had experimentally demonstrated classical interference between the generated subharmonic field in the OPA system and the injected coherent signal beam at the subharmonic frequency [18]. Here, we replace the coherent signal beam with a squeezed vacuum field generated in another OPO operated below threshold (labelled as OPO, as shown in Fig.1). Since the output field of the subthreshold OPO is a quadrature squeezed vacuum state, its relative phase to the amplification (or deamplification) phase of the OPA (Fig.1) has to be predetermined (by using a homodyne detection setup with a local oscillator beam) and controlled, which is quite different from the injected classical coherent signal beam [18]. We investigate the amplification and deamplification of both the squeezed and unsqueezed quadratures of the input quantum field, respectively, and show how such phase-sensitive amplifier can be used to modify and control the input squeezed states of light. The degree of squeezing can be improved by further deamplifying the input squeezed quadrature.

Our experimental setup is shown schematically in Fig.1. A diode-pumped intra-cavity frequency-doubled (continuous-wave ring $\mathrm{Nd}: \mathrm{YVO}_{4} / \mathrm{KTP}$ ) laser is used as the light sources, which provide about $200 \mathrm{~mW}$ of the second-harmonic light at $532 \mathrm{~nm}$ and $50 \mathrm{~mW}$ of the fundamental light at $1064 \mathrm{~nm}$ simultaneously. The secondharmonic light is divided into two parts to pump the OPO and the OPA systems respectively. The OPO and OPA systems have the same structure, each of which has a cavity composed of two coupling mirrors with same radius of curvature of $30 \mathrm{~mm}$. A $12 \mathrm{~mm}$ long PPKTP (periodically-poled KTP) crystal (Raicol Inc.) with antireflection-coated flat surfaces for both wavelengths is placed in each optical cavity. The reflectivities are $99.5 \%$ at $1064 \mathrm{~nm}$ and $60 \%$ at $532 \mathrm{~nm}$ for the input coupler $M 1$, which is mounted on a PZT to adjust the cavity length. The output coupler $M 2$ has a reflectivity of $93 \%$ for the OPO at $1064 \mathrm{~nm}$ (and 97\% for the OPA), and is a high reflector $(>99 \%)$ at $532 \mathrm{~nm}$. The coatings of the cavity mirrors also provide a second harmonic power build-up (few times) when the fundamental wavelength is on resonance with the cavity. The OPO (and the OPA) cavity length is $60 \mathrm{~mm}$. The temperature of the PPKTP crystal is actively controlled at millidegree Kelvin level around the operation temperature $\left(31.3^{\circ} \mathrm{C}\right)$ for optimizing the optical parametric down-conversion process at the cho- 


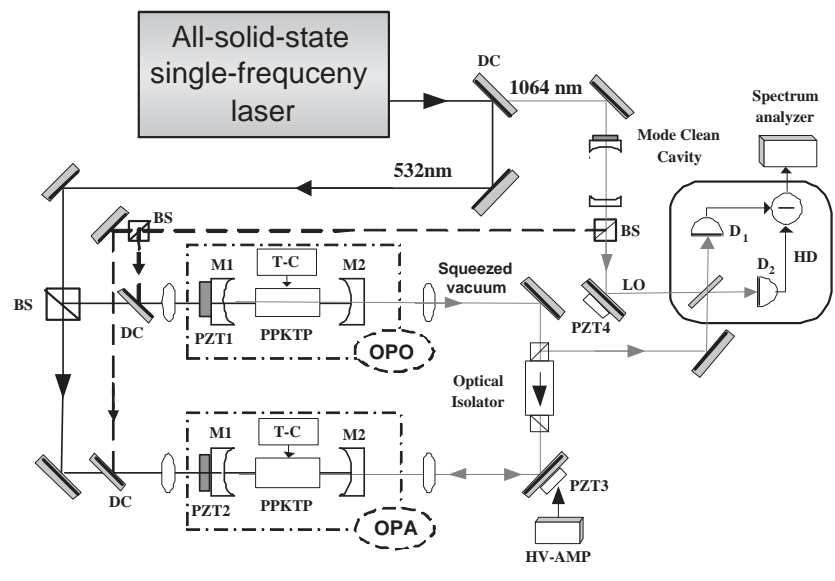

FIG. 1: Schematic of the experimental setup for quantum interference phenomena in the phase-sensitive OPA system inside the optical cavity with an injected squeezed vacuum state. A squeezed vacuum state is generated from the subthreshold OPO, then injected into the OPA as the input signal. DC: dichroic mirror; $\lambda / 2$ : half-wave plate; $D_{1}, D_{2}$ : detectors; T-C: temperature controller, HV-AMP: high voltage amplifier; PZT: piezoelectric transducer; BS: beam splitter; LO: local oscillator; HD: homodyne detection.

sen wavelength.

The OPA is pumped by a second harmonic beam with a wavelength of $532 \mathrm{~nm}$ through $M 1$, and the signal input beam is injected through the mirror $M 2$ from right. The signal field goes through the optical isolator before entering the OPA cavity. When it goes through the same optical isolator after reflecting from the OPA cavity, its polarization rotates 90 degrees from its original polarization axis, and is reflected by the polarization beam splitter cube. This reflected signal beam then combines with the local oscillator beam to be measured by the balanced homodyne detector [12], as shown in Fig.1. Before injecting the squeezed state into the OPA cavity, we first characterize the phase-sensitive optical amplifier by injecting a weak coherent signal beam at the subharmonic wavelength [18]. When the pump beam is blocked, the cavity reflected signal spectrum is directly detected by a photodetector (not using the homodyne detection setup), which is shown in Fig.2(a) as a simple Lorentzian profile. When the pump beam is turned on, but at a lower power (at $0.2 P_{t h}$, where $P_{t h}$ is the OPO threshold for this OPA+cavity system), the generated subharmonic field is either in-phase (amplifying) or out-phase (deamplifying) with the input coherent signal field (by tuning the phase of the injected signal beam with the mirror mounted on PZT3), as shown in Figs.2(b) and 2(c), respectively. As the pump power is increased to $0.5 P_{t h}$, the coherent signal is greatly amplified at the resonance when it is in-phase with the OPA amplifier (Fig.2(d)), but suppressed (deamplified) when it is out-phase with the OPA (Fig.2(e)). As one can see that shoulders appear for both

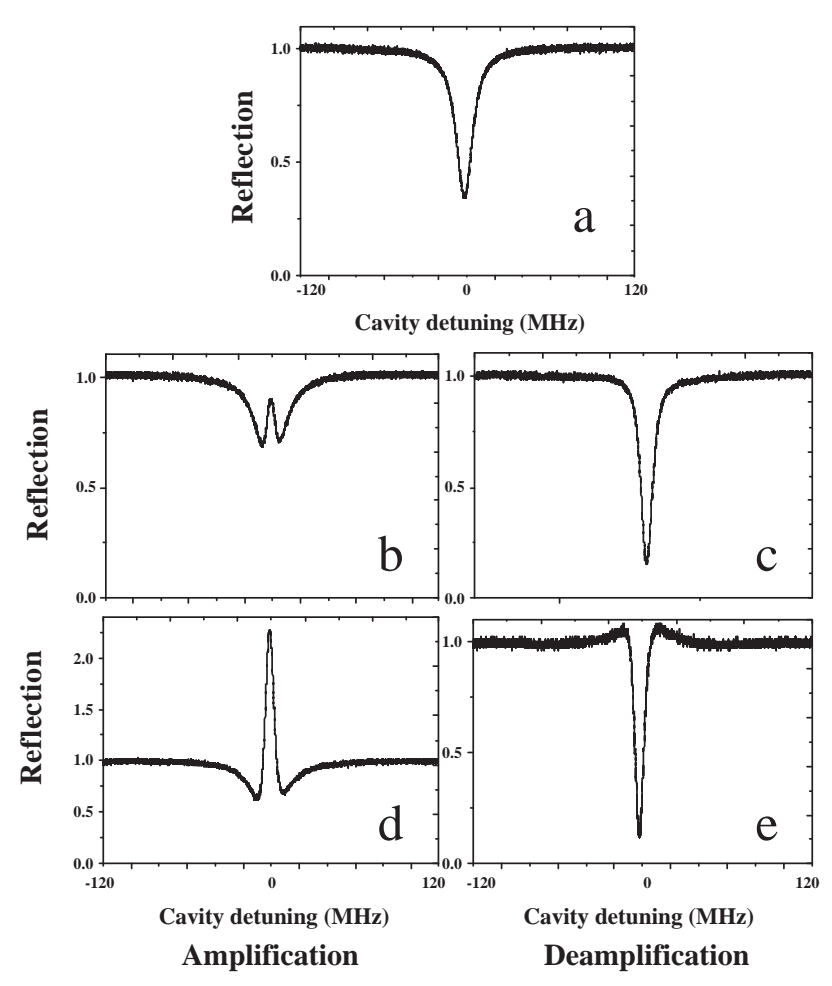

FIG. 2: The reflection spectra of the subharmonic field from the OPA cavity injected with the coherent signal beam, as a function of the cavity detuning for different pump powers. (a) without the pump beam; (b) and (c) operated in-phase (amplifying) and out-phase (deamplifying) at resonance between the injected signal beam and the pump beam, respectively, with the pump power at $0.2 P_{t h} ;(\mathrm{d})$ and (e) with the pump power at $0.5 P_{t h}$.

amplified and deamplified spectra just outside the resonant peak (dip), which indicate that small amplification can occur when the cavity is detuned from the resonance ( Fig.2(e)) even for the deamplified operation at resonance. Similarly, small deamplification can appear in the amplified phase for the OPA outside the resonance (Fig.2(d)). Notice that the amplified peak (and deamplified dip) has a narrower linewidth than the input coherent signal dip reflected from the "empty cavity" (Fig.2(a)) due to such deamplification shoulders in the amplification phase (and the same is true for the deamplifying phase with the amplification shoulders).

A quadrature squeezed vacuum state is generated from the subthreshold OPO. Due to the high nonlinear coefficient of the PPKTP crystal, the measured threshold power of the OPO is $P_{t h}=80 \mathrm{~mW}$. The OPO correlates the upper and lower quantum sidebands of a vacuum field that enters the OPO around the center frequency $\omega_{0}$. The correlation of the quantum sidebands appears as the squeezed vacuum field [19]. When pumped at frequency $2 \omega_{0}$ and operated at $40 \mathrm{~mW}$ below threshold, about 2 $\mathrm{dB}$ squeezing at the sideband frequency of $3.5 \mathrm{MHz}$ is 
detected, which passes through the optical isolator and is then injected into the OPA as the input signal. Since the squeezed vacuum state is elliptic in phase space, it has to be oriented relative to the amplification axis of the OPA system carefully. This can be done by tuning PZT3 and checked by detecting it with the local oscillator in the balanced homodyne detector. The injected squeezing vacuum field is reflected by the OPA cavity. When passing through the Faraday rotator again, the polarization plane of the backward light is rotated $45^{\circ}$ in the same direction as the initial tilt. This reflected light is then completely reflected by the polarizer just behind the Faraday rotator, combined with the local oscillator field from the laser at a 50/50 beam splitter, and detected by the balanced homodyne detector system. The local oscillator beam passes through a mode-cleaner cavity, so that it is in a similar beam profile as the reflected signal beam from the OPA cavity, and a fringe visibility of $\sim 96 \%$ between them has been achieved in the experiment.

First, let us look at the squeezed quadrature of the input signal beam as shown in the diagram below Fig.3(a) (e.g. $\theta=0$ ) by choosing the phase of the local oscillator (PZT4) relative to the input squeezed vacuum state. When the pump beam for the OPA is blocked, while fixing the phase between the local oscillator and the reflected vacuum squeezing, and scanning the length of the OPA cavity, the reflected squeezing signal spectrum is shown in Fig.3(a). The center part of the reflected spectrum is modified by the optical cavity with the middle (on resonance) barely below the shot-noise limit (SNL) (but above the degree of squeezing at far off resonance), which is used as a reference level for input squeezing. This spectral shape is induced by the absorption and dispersion properties of the "empty cavity" [20]. The OPA cavity, as the empty cavity in this case, is an over-coupled resonator for the subharmonic field due to $\gamma_{\text {out }}>\gamma_{i n}+\gamma_{l}$, where $\gamma_{l}, \gamma_{i n}$ and $\gamma_{\text {out }}$ are the decay rates of the subharmonic field resulting from internal losses, the input mirror M1, and the output mirror M2, respectively [21]. Next the pump beam for the OPA is turned on. The relative phase between the injected squeezing signal and the pump beam of the OPA is tuned by PZT3, which determines the amplification or deamplification of the signal beam at resonance. When the relative phase is tuned to be $\phi=\pi / 2$, the input squeezing quadrature is deamplified by the OPA with the degree of squeezing at the line center to be below the degree of squeezing at far detuning, as shown in Fig.3(c). This indicates a further squeezing by the OPA for the initial squeezed quadrature. As the phase of the signal is tuned in-phase with the pump beam $(\phi=0)$, the squeezed quadrature is amplified, which reduces the initial degree of squeezing and makes it above the SNL, as shown in Fig.3(e). The system is operated at a pump power of $0.5 P_{t h}$. The shoulders in the amplification and deamplification spectra just outside the resonant dip (or peak), as shown on Figs.2(d) and 2(e),

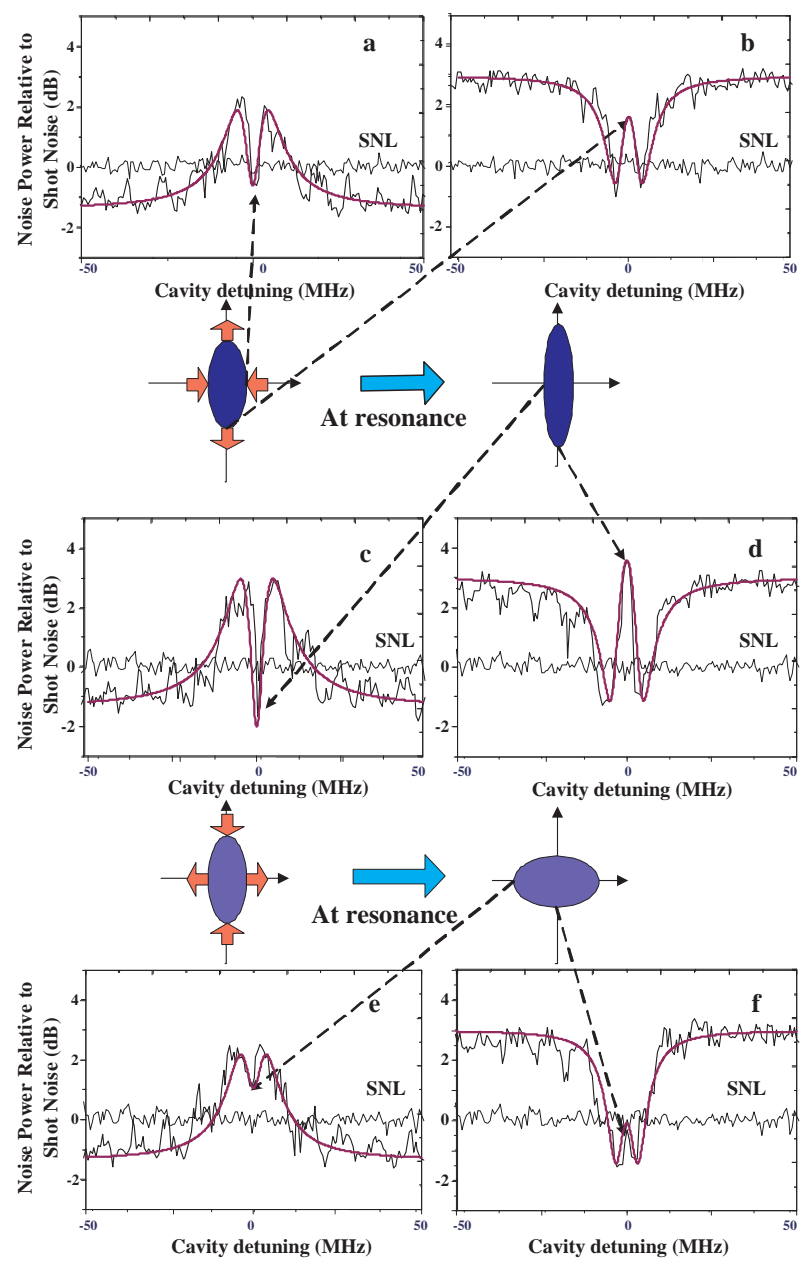

FIG. 3: (Color online). The reflection spectra of the subharmonic field from the OPA cavity injected with the squeezed vacuum field as a function of the cavity detuning. Noise levels are displayed as the relative power compared to the shot-noise limit. Quantum noise is measured at the sideband frequency of $3.5 \mathrm{MHz}$. (a) and (b) without the pump beam; (c),(d),(e) and (f) with pump power at $0.5 P_{t h}$. The curves plotted with the purple lines are the theoretical calculations.

also modify the reflected spectral shapes at the sides of the peaks in Figs.3(c) and 3(e).

Now let us examine the situation with the unsqueezed quadrature as the input signal (e.g. $\theta=\pi / 2$ ). Figure 3(b) shows the reflected signal spectrum without the pump beam for the OPA. As the pump beam is turned on and $\phi$ is set at $\phi=\pi / 2$, the deamplifiction for the squeezed quadrature (Fig.3(c)) becomes amplification for the unsqueezed quadrature, as shown in Fig. 3(d). The central peak is significantly amplified. As the signal phase is tuned $90^{\circ}$ relative to the phase of the pump beam $(\phi=0)$, the amplification for the squeezed quadrature (Fig.3(e)) becomes deamplification for the unsqueezed quadrature by the OPA (Fig.3(f)), where the unsqueezed central peak begins to fall below the SNL. The effect of 
the amplification shoulders in the deamplification phase can be seen more clearly here (Fig.3(f)) as the shape of the dip becomes sharper near the baseline (comparing to the dip in Fig.3(b)). To make quantitative theoretical comparisons with the above experimental results, the homodyne spectra for the amplitude and phase quandratures [22] are calculated with experimental parameters including all loss mechanisms. Our theoretically calculated results (purple lines) are plotted together with the experimental data in Fig. 3, which show excellent agreements. These theoretical curves can only be qualitatively compared with the results presented in Ref. [16], where power spectra were given without including any losses.

Figure 3(c) represents a case where the deamplification phase of the OPA is chosen relative to the squeezed quadrature of the input squeezed vacuum state. The degree of squeezing has been further improved below its input level, which can be used as a "squeezing amplifier" for applications in quantum communication and quantum information processing. In principle, the degree of squeezing for the input squeezed vacuum state can be increased further by improving the quality of the OPA+cavity system, such as reduction of internal losses, and operating the system closer to the OPO threshold, where the quantum nature of the generated field will be more pronounced [17]. There are two significant points coming out of this experiment: 1) Generating stronger squeezing by an OPA with an injected squeezed vacuum may improve Einstein-Podolsky-Rosen (EPR) entanglement further if we use two squeezed lights and a 50/50 beam splitter to produce the EPR entangled beam; 2) Injecting other quantum states into an OPA can lead to important advances in quantum information processing [10, 11, 12, 13, 14].

In summary, we have experimentally demonstrated quantum interferences between the input squeezed vacuum state and the quantum field generated in the OPA system at different quadrature phases. We have shown the manipulations of quantum fluctuations due to quantum interferences by the OPA inside an optical cavity, as predicted by Agarwal [16]. Our experiment also indicates that the input squeezing can be further enhanced to a higher degree when appropriate relative phase is chosen between the input quantum signal field and the pump field for the OPA. Such manipulations of quantum fluctuations by a phase-sensitive optical amplifier (a quantum state generator itself) are essential in quantum information processing and quantum networking [23].

†Corresponding author's email address: jzhang74@yahoo.com, jzhang74@sxu.edu.cn

J. Zhang thanks K. Peng and C. Xie for the helpful discussions. This research was supported in part by NSFC for Distinguished Young Scholars (Grant No. 10725416), National Basic Research Program of China (Grant No. 2006CB921101), NSFC Project for Excellent Research Team (Grant No. 60821004), National Natural Science
Foundation of China (Grant No. 60678029), Doctoral Program Foundation of the Ministry of Education, China (Approval No. 20050108007), the PCSIRT (Grant No. IRT0516), the TYMIT and TSTIT of Shanxi.

[1] D. Akamatsu, K. Akiba, and M. Kozuma, Phys. Rev. Lett. 92, 203602 (2004).

[2] D. Akamatsu, et al., Phys. Rev. Lett. 99, 153602 (2007).

[3] M. Arikawa, et al., Opt. Express 15, 11849 (2007).

[4] G. Hetet, et al., arXiv:0803.2097 [quant-ph].

[5] K. Honda, et al., Phys. Rev. Lett. 100, 093601 (2008).

[6] J. Appel, E. Figueroa, and D. Korystov, M. Lobino, and A. I. Lvovsky, Phys. Rev. Lett. 100, 093602 (2008).

[7] S. E. Harris, Phys. Today 50, 37 (1997); J. P. Marangos, J. Mod. Opt. 45, 471 (1998); M. Fleischhauer, A. Imamoglu, J. P. Marangos, Rev. Mod. Phys. 77, 633 (2005).

[8] L. M. Duan, M. D. Lukin, J. I. Cirac, P. Zoller, Nature 414, 413 (2001).

[9] C. Simon, et al., Phys. Rev. Lett. 98, 190503 (2007).

[10] A. Lamas-Linares, C. Simon, J. C. Howell, and D. Bouwmeester, Science 296, 712 (2002); F. De Martini, et al., Phys. Rev. Lett. 92, 067901 (2004). F. De Martini, et al., Phys. Rev. Lett. 95, 240401 (2004).

[11] G. M. D'Ariano, F. De Martini, and M. F. Sacchi, Phys. Rev. Lett. 86, 914 (2001); S. L. Braunstein, et al., Phys. Rev. Lett. 86, 4938 (2001); J. Fiurasek, Phys. Rev. Lett. 86, 4942 (2001).

[12] F. De Martini, V. Buk, F. Sciarrino, and C. Sias, Nature (London) 419, 815 (2002).

[13] F. De Martini, et al., arXiv:0806.2218 quant-ph].

[14] T. C. Ralph, Opt. Lett., 24, 348 (1999).

[15] R. Bruckmeier, K. Schneider, S. Schiller, and J. Mlynek, Phys. Rev. Lett. 78, 1243 (1997).

[16] G. S. Agarwal, Phys. Rev. Lett. 97, 023601 (2006).

[17] L. A. Wu, M. Xiao, H. J. Kimble, J. Opt. Soc. Am. B 4, 1465 (1987); K. Schneider, M. Lang, J. Mlynek, S. Schiller, Opt. Express 2, 59 (1996); H. Vahlbruch, et al. Phys. Rev. Lett. 100, 033602 (2008); Y. Takeno, M. Yukawa, H. Yonezawa, A. Furusawa, Opt. Express 15, 4321 (2007).

[18] H. Ma, C. Ye, D. Wei, and J. Zhang, Phys. Rev. Lett. 95, 233601 (2005); C. Ye and J. Zhang, Phys. Rev. A 73, 023818(2006); C. Ye and J. Zhang, Opt. Lett. 33, 1911 (2008).

[19] J. Zhang, Phys. Rev. A 67, 054302 (2003).

[20] M. D. Levenson, R. M. Shelby, S. H. Perlmutter, Opt. Lett. 10, 514 (1985); P. Galatola, et al., Opt. Commun. 85, 95 (1991); J. Zhang, et al., J. Opt. Soc. Am. B 17, 1920 (2000); A. Zavatta, F. Marin, G. Giacomelli, Phys. Rev. A 66, 043805(2002).

[21] B. J. J. Slagmolen, M. B. Gray, K. G. Baigent, D. E. McClelland, Appl. Opt. 39, 3638 (2000).

[22] D. F. Walls, G. J. Milburn, Quantum Optics, SpringerBerlag, Berlin, 1995; K Wang, A. Sinatra, L. A. Lugiato, Eur. Phys. J. D 12, 339 (2000).

[23] S. L. Braunstein, P. van Loock, Rev. Mod. Phys. 77, 513 (2005). 